\title{
Quelques pistes pour gagner le pari de l'Éducation au Développement Durable au Chili
}

Adolfo Berrios et Enrique Martinez

\section{(2) OpenEdition}

Édition électronique

URL : http://journals.openedition.org/ere/801

DOI : $10.4000 /$ ere.801

ISSN : 2561-2271

Éditeur

Centr'ERE

\section{Édition imprimée}

Date de publication : 20 décembre 2016

ISSN : 1373-9689

\section{Référence électronique}

Adolfo Berrios et Enrique Martinez, «Quelques pistes pour gagner le pari de l'Éducation au

Développement Durable au Chili », Éducation relative à l'environnement [En ligne], Volume 13 - 2 | 2016, mis en ligne le 20 décembre 2016, consulté le 21 février 2020. URL : http://journals.openedition.org/ ere/801 ; DOI : 10.4000/ere.801 


\title{
Quelques pistes pour gagner le pari de l'Éducation au Développement Durable au Chili
}

\author{
Adolfo Berrios et Enrique Martinez
}

1 Depuis sa gestation au sein des organisations internationales, la notion de Développement Durable (DD) est devenue peu à peu un enjeu majeur pour les systèmes éducatifs. Face à la dégradation des écosystèmes provoquée par les activités humaines, mais sans renoncer au développement économique, les Nations Unies ont décidé de faire du DD le modèle à suivre pour l'ensemble des pays de la planète. Compte tenu de la place qu'occupe l'éducation pour rendre opérationnelle cette notion, l'UNESCO a pris en charge pendant les années 2005-2014 la décennie de l'Éducation au Développement Durable (EDD) et exhorté les États membres à mettre en place des stratégies nationales afin d'atteindre les buts poursuivis par ce projet onusien.

2 Historiquement attribuée au rapport Brundtland, le DD est souvent réduit à l'énoncé suivant : « un développement qui répond aux besoins du présent sans compromettre la capacité des générations futures de répondre aux leurs » (Brundtland, 1987/trad. 1989). Au cours des ans, l'interprétation de cet énoncé a forgé une image lisse du DD qui a contribué à sa promotion et publicité. En effet, ce modèle est représenté par un schéma de trois cercles, où chacun symbolise les trois piliers principaux du projet: l'environnemental, le social et l'économique. Selon ce schéma, le DD se présente comme le point de rencontre des trois cercles, suggérant ainsi un modèle de développement à la fois capable de satisfaire les besoins de la population, réduire les inégalités sociales et préserver l'environnement. Ce schéma cache cependant certaines ambiguïtés conceptuelles. Latouche (2006) considère qu'il s'agit plutôt d'un «oxymore conceptuel », c'est-à-dire, une sorte de bricolage de mots et de discours disparates qui tentent de concilier la croissance économique avec la protection des écosystèmes. Grâce à ce flou conceptuel, le DD a rassemblé sous la même bannière les défenseurs de l'écologie et de la croissance. Selon Rist (2012), les bonnes intentions du rapport 
Brundtland ont contribué à diffuser le paradigme occidental du développement, qui fait de la croissance économique l'idéal et la base des économies nationales.

3 C'est sous cet angle et dans cette perspective que le projet de réaliser un développement durable pour le $\mathrm{XXI}^{\text {e }}$ siècle s'est installé au Chili. Le panorama socioéconomique que présentait le pays, en raison des réformes économiques néolibérales introduites au début des années quatre-vingt, s'emboîtait parfaitement avec le discours préconisé par l'Organisation des Nations Unies (ONU). En prenant l'exemple de l'adoption et l'adaptation de l'EDD au Chili, nous questionnerons ici l'objet central de cette éducation, le DD, et montrerons comment celui-ci a été instrumentalisé par le système éducatif du pays. Notre analyse révèlera qu'au cours de ce processus, le DD a fait l'objet de détournements idéologiques, qui ont transformé le système éducatif dans un laboratoire qui consolide plutôt un modèle peu "durable ». Ce constat nous oblige à mettre en œuvre de toute urgence une approche critique, afin que les élèves prennent position en tant que futurs citoyens et puissent prendre part aux défis qu'entraine ce projet de développement.

\section{La déclinaison de l'EDD au Chili}

4 Le processus qui accompagne l'intégration des objectifs voulus par le projet du DD au Chili se place dans un contexte national particulier. D'un point de vue historique, au début des années quatre-vingt-dix, le pays retrouve la démocratie après dix-sept ans de dictature militaire (1973-1990). Avec le redémarrage du système démocratique, les nouvelles autorités politiques du pays doivent affronter un système économique interne en pleine mutation, en raison des réformes néolibérales menées pendant cette période. Ces réformes économiques, qui accordent à l'État un rôle subsidiaire, auront comme conséquence la privatisation de la plus grande partie de l'appareil social, ce qui va avoir un impact profond sur le mode de financement et de gestion des systèmes de retraite, de santé et d'éducation.

5 Le système éducatif sera précisément l'un des principaux chantiers de ces réformes. Selon Cox (2003), la mise en œuvre de ces transformations aura pour effet de décentraliser l'administration économique du ministère, moyennant l'introduction de nouveaux outils liés au marché, comme les subventions à la demande. Dans ce nouveau cadre, qui permettra dorénavant la participation du privé dans la gestion d'établissements scolaires du pays, le statut de l'État dans le domaine de l'éducation nationale est redéfini et une partie de ses fonctions administratives est transférée aux pouvoirs municipaux. Bien que l'élaboration des programmes d'études des différentes disciplines scolaires reste aux mains du Ministère de l'Éducation (MINEDUC), cette nouvelle étape de l'éducation chilienne donne lieu à une "flexibilisation curriculaire ", permettant à chaque établissement d'accepter ou de modifier les programmes selon ses besoins.

6 C'est dans ce contexte que le DD a été intégré par les nouvelles autorités démocratiques dans l'ensemble du système éducatif du pays. Dans le cas du Chili, comme dans celui d'autres pays latino-américains, le discours sur le DD qui s'impose à la suite de la Conférence de Rio de 1992, a englouti une mosaïque d'expériences liées à l'éducation à l'environnement (Gonzalez-Gaudiano, 2001). Ainsi, dans ce processus de recadrage en faveur du DD, plusieurs ONG, ainsi que la Comisión Nacional del Medio Ambiente $(\text { CONAMA })^{1}$, récupèrent cette notion afin de réorienter leurs activités éducatives en 
matière de protection de l'environnement. De manière parallèle, cette notion apparaît pour la première fois comme un objectif transversal dans les nouveaux programmes $\mathrm{du}$ primaire et du secondaire, confirmant ainsi la place qu'occupe dorénavant le DD. Pour répondre à la nouvelle vague des prescriptions internationales en faveur du projet que représente l'EDD, l'État se dote en 2009 d'une stratégie nationale. Cette Política Nacional de Educación para el Desarrollo Sustentable (PNEDS) affirme que l'éducation doit promouvoir une citoyenneté active dans la construction du DD dans le pays, et souligne en même temps que ce mode de développement est la manière de concilier le développement économique et social avec la préservation de l'environnement.

Bien que la PNEDS accorde un rôle prioritaire à l'éducation, la mise en œuvre de l'EDD se heurte à un contexte de gouvernance particulier, qui donne un caractère diffus aux objectifs voulus par l'UNESCO. Avec un MINEDUC affaibli dans son rôle de preneur de décisions en matière éducationnelle, les vrais exécutants de l'EDD dans le système éducatif chilien s'avèrent être les ministères de l'Énergie et de l'Environnement. Ce sont essentiellement les programmes País de eficiencia energética ${ }^{3}$ et Sistema Nacional de Certificación Ambiental de Establecimientos Educacionales ${ }^{4}$, qui pendant cette décennie ont travaillé sur les objectifs des plans d'études ${ }^{5}$ élaborés par le MINEDUC. En mettant l'accent sur la responsabilité individuelle moyennant l'apprentissage de pratiques sociales, ces deux programmes semblent s'éloigner des problématiques soulevées par la mise en place des projets défendant une optique de DD dans le pays. C'est le cas, par exemple, de l'exploitation des ressources minières et leur consommation d'énergie. Effectivement, les conséquences de ces activités minières provoquent divers conflits socio-environnementaux qui mettent en évidence le faible contrôle de l'État, tant du point de vue fiscal qu'en ce qui concerne la gestion des déchets (Orellana et coll., 2008; Folchi, 2003).

8 Par ailleurs, si on examine la réalité du système éducatif pendant ces deux dernières décennies, l'éducation chilienne est loin d'atteindre le rôle de transformation sociale attribué par le DD à l'école. En effet, le réaménagement du rôle de l'État dans l'éducation va provoquer une énorme brèche dans la qualité et l'équité des apprentissages des élèves dans les différents établissements du système scolaire. Malgré les réformes des années 90 qui ont accru l'investissement public en matière d'éducation, le rôle de l'État n'a pas été modifié et le marché a continué à être le seul régulateur du système (Brunner et Cox, 1995 ; Cox et Lemaitre, 1999). Ainsi, comme le signale le rapport DEMRE (2010) concernant les résultats du baccalauréat de l'année 2009, seuls deux lycées publics figurent parmi les cent premiers et ce sont les établissements ${ }^{6}$ privés et payants qui obtiennent les meilleurs résultats. En tenant compte que ces derniers établissements représentent le $9 \%$ du total des établissements scolaires chiliens, le système éducatif agit donc plutôt comme un générateur d'inégalités, en reproduisant les différences socio-économiques de la société chilienne. Reflet de ces différences, l'index d'inégalité des salaires, le coefficient de $\mathrm{Gini}^{7}$, est très élevé au pays et jusqu'à ce jour, le Chili est resté l'un des pays les plus inégaux selon l'Organisation de Coopération et de Développement Économique (OCDE, 2013).

9 Étant donné le contexte décrit ci-dessous, nous nous interrogeons sur les réelles possibilités qu'offre l'éducation chilienne pour devenir un agent de transformation sociale. En effet, les textes des organisations internationales insistent sur le fait que pour concrétiser le DD : « il faut disposer d'outils pédagogiques plus complets, centrés sur les compétences relatives à la pensée critique et à la prise de décision rationnelle, 
afin de donner aux citoyens la capacité d'appréhender les problèmes les plus complexes qui se posent aux communautés et aux nations en termes de durabilité » (UNESCO, 2009 , p. 10). Il nous semble que c'est moyennant une nouvelle grille de lecture que cette notion pourrait contribuer à transformer cette réalité.

\section{La nécessité de tendre vers une approche critique de I'EDD au Chili}

10 Il nous parait que c'est à travers une approche critique que la notion de DD devrait être réappropriée par le système éducatif chilien. Selon Sauvé (2005), la question centrale qui caractérise cette approche est celle du "pourquoi ?». Dans cette perspective, il s'agit de décortiquer les réalités socio-environnementales afin de remettre en question les fondements, les intentions et les valeurs sous-jacentes qui entourent la relation personne-société-environnement. Sous cet angle d'approximation, les apprentissages des élèves dans le cadre de l'EDD pourraient être enrichis. En effet, jusqu'à ce jour, la plupart des initiatives mises en place portent sur des thématiques environnementales et mettent l'accent sur l'apprentissage de bonnes pratiques. Ceci n'est pas suffisant pour comprendre la complexité des défis que soulèvent les projets liés au DD. Afin de favoriser une citoyenneté engagée à l'égard des impacts économiques, sociaux et environnementaux que suscite l'exploitation des ressources du pays, l'éducation chilienne doit privilégier le développement de nouvelles compétences.

11 En tenant compte que le flou conceptuel de la notion de durabilité sert d'alibi pour les intérêts des lobbies économiques, l'exercice d'une vigilance critique face aux promoteurs du DD apparaît comme une priorité pour les acteurs du monde de l'éducation chilienne. Dans ce sens, Sauvé (2013) signale que le développement de compétences d'ordre critique, éthique et politique pourrait contribuer à mieux s'emparer de ce type de situations et de prendre du recul face aux idéologies qui instrumentalisent la société civile. En mobilisant des connaissances, des habiletés cognitives, des attitudes et des valeurs, ces compétences peuvent contribuer à forger une écocitoyenneté consciente, responsable et participative par rapport aux enjeux du DD. Pour ce faire, «il importe entre autres de valoriser et de soutenir l'apprentissage social dans les divers contextes d'interaction citoyenne et d'ancrer la formation dans les réalités des milieux de vie » (Sauvé, 2013, p. 21). C'est précisément là qu'à notre avis se trouvent les clés pour élargir vers une perspective critique et citoyenne l'EDD au Chili. Effectivement, dans un pays où le discours du développement, synonyme de la croissance ou du progrès économique est dominant, un regard critique et réflexif sur les contradictions du DD pourrait encourager les acteurs du monde de l'éducation à remettre en question ce modèle de développement.

Dans le contexte d'une éducation critique, la perspective didactique des Questions Socialement Vives (QSV) offre des balises théoriques et méthodologiques pour favoriser ce type de raisonnement dans les pratiques d'enseignement-apprentissage. En considérant les questions d'actualité qui ont un impact sur l'école, comme ce serait le cas des problématiques que soulève le DD, Legardez et Simonneaux (2011) suggèrent qu'une telle conjoncture oblige à mettre en œuvre une analyse socio-épistémologique des savoirs impliqués. En effet, les QSV entremêlent des enjeux politiques, idéologiques, scientifiques et de valeurs qu'il est nécessaire de prendre en compte lorsque ces types de questions sont abordées en classe. En mettant l'accent sur la capacité à argumenter 
et à débattre, leur but est de mieux comprendre ces controverses, afin que les élèves prennent conscience des défis de ce modèle et soient capables de prendre décisions (Simonneaux, 2006). Face à l'injonction planétaire de l'EDD, la recherche dans le champ de QSV se révèle aujourd'hui fondamentale pour savoir pourquoi et comment les acteurs éducatifs de différents pays s'approprient, d'un point de vue de leurs représentations sociales, de l'objet central de cette éducation. À ce stade, ces recherches ont permis d'établir un premier bilan par rapport aux enjeux que soulève l'enseignement des questions associées au DD et les obstacles liés aux divers contextes sociohistoriques et culturels qui freinent la compréhension de cette question complexe (Jeziorski et Legardez, 2013 ; Barthes Jeziorski, 2012 ; Floro, 2011 ; Freudigier, 2010).

Dans le contexte d'une éducation critique, la perspective didactique des Questions Socialement Vives (QSV) offre des balises théoriques et méthodologiques pour favoriser ce type de raisonnement dans les pratiques d'enseignement-apprentissage. En considérant les questions d'actualité qui ont un impact sur l'école, comme ce serait le cas des problématiques que soulève le DD, Legardez et Simonneaux (2011) suggèrent de mettre en œuvre une analyse socio-épistémologique des savoirs impliqués. En effet, les QSV entremêlent des enjeux politiques, idéologiques, scientifiques et de l'ordre des valeurs qu'il est nécessaire de prendre en compte lorsque ce type de questions est abordé en classe. En mettant l'accent sur la capacité à argumenter et à débattre, le but est de mieux comprendre ces controverses, afin que les élèves prennent conscience des défis soulevées par ces questions et soient capables de prendre décisions (Simonneaux, 2006). Face à l'injonction planétaire de l'EDD, la recherche dans le champ de QSV s'avère aujourd'hui fondamentale pour examiner pourquoi et comment les éducateurs et formateurs de différents pays s'approprient, du point de vue de leurs représentations sociales, de l'objet central de cette éducation au développement durable. Jusqu'ici, certaines recherches ont permis d'établir un premier bilan des enjeux que soulève l'enseignement des questions associées au DD et des obstacles liés aux divers contextes sociohistoriques et culturels qui freinent la compréhension de cette question complexe (Jeziorski et Legardez, 2013; Barthes Jeziorski, 2012 ; Floro, 2011 ; Freudigier, 2010).

En ce sens, une approche didactique sous l'angle de QSV, pourrait aider les enseignants chiliens à incorporer dans leurs pratiques éducatives les controverses et contradictions véhiculées par le projet mondial de développement durable. En prenant en compte la nature de savoirs de références, instables et incertaines, cette perspective didactique invite à réfléchir sur la complexité théorique et pratique qu'entraine le vernis «durable " que l'ONU tente d'imposer à l'actuel modèle économique de la planète. Ainsi, ce courant didactique des QSV pourrait favoriser le développement de véritables compétences citoyennes, qui au-delà de la réalisation d'écogestes, contribuent à développer la capacité de dialogue et de discussion à propos des enjeux qui pose le DD au pays.

15 C'est donc plutôt sous l'angle du réveil de l'esprit critique à propos des questions soulevées par le DD que doit se réorienter le chantier que la PNEDS tente de mettre en œuvre dans le pays. Pour l'instant, l'éducation chilienne est en train de perdre le pari de l'EDD en institutionnalisant une idée utilitariste de la durabilité ${ }^{8}$, qui voit dans la maitrise des ressources naturelles la clé du bonheur économique. Face au danger d'endoctrinement qu'implique ce credo, le système éducatif doit se doter, plus que jamais, de pratiques éducatives critiques et citoyennes. Pour ce faire, l'éducation 
chilienne doit fournir aux élèves les compétences qui leur permettent d'aborder les controverses que soulève le DD, afin qu'ils puissent devenir les acteurs et gestionnaires d'un projet de développement approprié. En écartant les contradictions et ambiguïtés qui entourent la notion de DD, il semble difficile de faire comprendre aux élèves qu'il s'agit d'un projet qui est toujours soumis à discussion et qui par conséquent, exige une démarche citoyenne critique et active, afin de prendre position face aux enjeux que pose la mise en place de tout programme qui porte l'étiquette du DD.

\section{Conclusion}

La mise en œuvre de l'EDD au Chili semble illustrer la prévalence des intérêts économiques par rapport aux questions socio-environnementales (Berrios, 2014). Mise au service des maximes néolibérales, l'éducation est le miroir d'un modèle qui, dans la vie quotidienne des Chiliens, ne répond pas à l'idée de durabilité proclamée par les discours des Nations Unies. Néanmoins, c'est dans la mise au jour des contradictions que génère ce modèle que se trouvent les pistes qui pourraient permettre au Chili de gagner le pari de l'EDD. En effet, au cours des cinq dernières années, divers mouvements sociaux centrés sur la défense de l'environnement ${ }^{9}$ et la qualité de l'éducation ${ }^{10}$ ont ouvert un débat national sur les politiques publiques menées dans ces deux domaines. La prise en compte du discours critique de ces mouvements offre une belle occasion de remettre en question l'image consensuelle du DD que la PNEDS est en train de construire et de souligner que ce ne sont pas seulement les sujets économiques qui préoccupent la société chilienne. Il importe de prendre en compte les implications sociales, politiques, environnementales et culturelles d'un tel projet de développement.

En considérant le contexte institutionnel du pays, reste à savoir si le MINEDUC a les moyens de répondre à ce défi, notamment, par l'élaboration d'un curriculum approprié et par la formation initiale et continue des enseignants.

\section{BIBLIOGRAPHIE}

Barthes, A. et Jeziorski, A. (2012). What Kind of Critical University Education for Sustainable Development? A Comparative Study of European Students and Social Representations. Journal of Social Science Éducation, 11 (4), 62-77.

Berrios, A., (2014). Des savoirs disciplinaires dans l'élaboration d'une représentation sociale du développement durable chez les élèves chiliens? Revue francophone du développement durable, 4 , 66-79.

Brundtland, H. G. (1989). Notre avenir à tous. Rapport de la Commission mondiale sur l'Environnement et le Développement. Montréal : Les Editions du Fleuve. [Trad. française de «Our common future » (1987)]. 
Brunner, J.J.et Cox, C. (1995). Dinámicas de transformación en el sistema educacional en Chile. Dans Puryear J. et Brunner J.J. (dir.), Educación, equidad y competitividad económica en las Américas (p. 101-152). Washington D.C. : OEA.

Cox, C. (2003). Las políticas educacionales en Chile en las últimas dos décadas del siglo XX. Dans Cox C. (dir.), Políticas educacionales en el cambio de siglo. La reforma del sistema escolar de Chile. (p. 19-113). Santiago : Editorial Universitaria.

Cox, C. et Lemaitre, M.J. (1999). Market and state principales of reform in chilean education. : policies and results. Dans Leiptziger D. et Perry G. (dir.), Chile : policy lessons and emerging challenges (p. 149-188). Washington D. C. : World Bank.

DEMRE (2010). Resultados proceso de admision. Récupéré du site : http://www.psu.demre.cl/ estadisticas/documentos-2010

Floro, M. (2011). Développement durable et questions socialement vives. Une approche territorialisée du discours enseignant. Dans Legardez A. et Simonneaux L. (dir.), Développement durable et autres questions d'actualité. Les Questions Socialement Vives dans l'enseignement et la formation (p. 163-180). Dijon : Éditions Educagri.

Folchi, M. (2003). La insustentabilidad del "boom minero" chileno : política y medio ambiente, 1983-2003. Ecología política, $n^{\circ} 26$, Barcelona, 23-49.

Freudiger, N. (2010). Comparaison de représentations d'élèves suisses et indiens sur le développement durable et le réchauffement climatique. Actes du congrès de l'Actualité de la recherche en éducation et en formation (AREF). Genève. Récupéré du site : https://plone2.unige.ch/ aref2010.

Gonzalez-Gaudiano, E. (2001). Otra lectura a la historia de la educación ambiental en América Latina y el Caribe. Desenvolvimento e Meio Ambiente, $n^{\circ}$. 3, 141-158

Jeziorski, A. et Legardez, A. (2013). Spécificités disciplinaires de l'éducation au développement durable dans les représentations des futurs enseignants français des sciences de la nature et des sciences humaines et sociales. Éducation Relative à l'Environnement : Regards - Recherches - Réflexions, $11,175-194$.

Latouche, S. (2006). Le pari de la décroissance. Paris : Fayard.

Legardez, A. et Simonneaux, L. (2011). Développement durable et autres questions d'actualité. Questions socialement vives dans l'enseignement et la formation. Dijon : Edition Educagri.

Ley General de Educación (2009). Récupéré du site : www.leychile.cl/Navegar?

idNorma $=269001 \&$ buscar $=$ general + de + educacion

Ley Bases generales del medio ambiente (1994). Récupéré du site : www.leychile.cl/Navegar? idNorma $=30667$

Martínez, E. A., Bazile, D., Thomet, M., Delatorre, J., Salazar, E., Leon-Lobos, P., Von Baer, I., Nuñez, L. (2010). Neo-liberalism in Chile and its impacts on agriculture and biodiversity conservation : the experience with the re-start of quinoa crop cultivation. Innovation and Sustainable Development. ISDA, 28 julio-1ํJ Julio 2010. Montpellier, Francia. Récupéré du site : http://hal.archives-ouvertures.fr/ ISDA2010

OCDE (2013). Regards sur l'éducation 2013- Les indicateurs de l'OCDE. Éditions OCDE. Récupéré du site : http://dx.doi.org/10.1787/eag-2013-fr

Orellana, I., Sauvé, L., Marleau, M.E., Labraña, R. (2008). La recherche critique en éducation relativement à l'environnement au sein du mouvement de résistance sociale face au projet 
minière de Pascua Lama. Éducation relative à l'Environnement : Regards - Recherches - Réflexions, volume 8, 23-47.

Politica nacional de Education para el Desarrollo sustentable (2009), Gobierno de Chile, Cuidando Chile Nueva Institucionalidad Ambiental. Récupéré du site : http://www.sinia.cl/1292/ articles-46509_recurso_2.pdf

Rist, G. (2012). Le développement : histoire d'une croyance occidentale. Paris : Presses de la Fondation nationale des sciences politiques.

Sauvé, L. (2005) Repères pour la recherche en éducation relative à l'environnement. Dans Sauvé, L., Orellana I., et Van Steenberghe, É. Éducation et Environnement - Un croisement de savoirs. Collection Les Cahiers scientifiques de l'ACFAS (Association francophone pour le savoir). 140. Montréal : Fides.

Sauvé, L. (2013). Au cœur des questions socio-écologiques : des savoirs à construire, des compétences à développer. Éducation relative à l'Environnement : Regards - Recherches - Réflexions, volume 11, 19-40.

Simonneaux, L. (2006). Quel enjeu éducatif pour les questions biotechnologiques. Dans A. Legardez A., \& Simonneaux L. (dir.), L'école à l'épreuve de l'actualité. Enseigner les questions socialement vives (p. 33-60). Paris : ESF.

\section{NOTES}

1. Créée par la loi Bases Generales del medio ambiente (1994), cette institution se transforme en 2009 en ministère de l'Environnement. Depuis sa création, une de ses fonctions est l'élaboration et la diffusion de programmes d'éducation environnementale.

2. Politique nationale d'éducation pour le développement durable.

3. Pays d'efficience énergétique. Ce programme du ministère de l'Énergie vise à développer des attitudes quotidiennes favorables à une utilisation efficiente et responsable de l'énergie.

4. Système national de certification environnementale des établissements scolaires. Ce programme du ministère de l'Environnement vise à prendre conscience de l'importance du développement durable dans la protection de l'environnement et du patrimoine naturel.

5. Il s'agit principalement des programmes d'études des disciplines d'Histoire, Géographie et Sciences sociales et de Sciences Naturelles qui sont considérées, d'un point de vue curriculaire, comme les responsables de l'EDD selon la PNEDS.

6. Il y a trois types d'établissements scolaires dans le système éducatif chilien : municipal, subventionné et privé.

7. Le coefficient de Gini mesure la disproportion entre les salaires. Ce coefficient varie de 0 à 1 , soit de l'égalité complète des salaires à une inégalité maximale. Selon Martinez et al. (2010), la une valeur était de 0,55 dans les années soixante.

8. Dans les principes qui inspirent l'élaboration de la Ley General de Educación (2009), la durabilité est considérée comme le respect de l'environnement et l'utilisation rationnelle des ressources naturelles.

9. Il s'agit du mouvement «Patagonie sans barrages ", organisé pour s'opposer au projet Hidroaysen qui cherche à construire cinq centrales hydroélectriques dans l'extrême sud du pays. 10. Depuis 2010, différentes manifestations ont rassemblé les étudiants, les professeurs et les organisations syndicales pour dénoncer le faible rôle de l'État dans le secteur de l'éducation. Bien que la réforme initiée par le gouvernement de Michelle Bachelet (2014-2018) a tenté de réguler le profit lucratif du secteur privé dans l'éducation, la qualité de l'éducation et les moyens pour y parvenir ne font pas, pour l'instant, l'objet de discussion dans la sphère politique nationale. 
INDEX

Index géographique : Chili

Mots-clés : éducation au développement durable

Keywords : education for sustainable development

\section{AUTEURS \\ ADOLFO BERRIOS}

Doctorant en sciences de l'éducation à l'Université d'Aix-Marseille, sa thèse repose sur comparaison des pratiques d'enseignement liées à l'Éducation au développement durable en France et au Chili. Professeur d'Histoire et Géographie, il a travaillé pendant six dans l'enseignement secondaire chilien.

\section{ENRIQUE MARTINEZ}

Docteur en biologie avec 30 ans d'expérience dans la recherche en écologie végétale des milieux marins et terrestres, il travaille depuis 2012 en collaboration scientifique avec le laboratoire d'Apprentissage, Didactique, Évaluation et Formation (ADEF) de l'Université d'Aix-Marseille. 